Amigo, S., Smith, A., \& Ludwig, T. (2008). Using Task Clarification, Goal Setting, and Feedback to Decrease Table Busing Times in a Franchise Pizza Restaurant. Journal of Organizational Behavior Management, 28(3), 176-187. DOI: 10.1080/01608060802251106 Version off record published by Published by Haworth / Taylor \& Francis and is available online at: http://www.informaworld.com/ (ISSN: 0160-8061)

Keywords: Task clarification |feedback | goal setting

\title{
Using Task Clarification, Goal Setting, and Feedback to Decrease Table Busing Times in a Franchise Pizza Restaurant
}

\author{
Seth Amigo, Andrew Smith, and Timothy Ludwig
}

\begin{abstract}
The current study investigated the effects of task-clarification, and manager verbal and graphic feedback on employee busing times at a pizza restaurant. Using an ABC design, taskclarification was provided in a memo, which described the process, priority, and goal time of busing. The busing time decreased slightly, from an average of 315 seconds during baseline to an average of 284 seconds after the task clarification memo. The majority of this change was accounted for by servers decreasing the time it took them to arrive at the table to begin busing. Subsequent to the employee memo, group feedback was administered in the form of verbal feedback and a weekly graph of average server busing times. This feedback was associated with reductions in busing time to an average 152 seconds. Changes in the feedback phase were caused almost exclusively by reductions in the time it took to clean the table once the server was there. A reversal to near-baseline busing times was observed in a follow-up phase. Prebusing and cross-busing were not associated with shorter busing times.
\end{abstract}




\section{ARTICLE}

In high volume restaurants, managers often have a difficult time reinforcing and maintaining correct cleaning behaviors, particularly with the waitstaff of the restaurant. This difficulty often arises because there are so many other competing behaviors that interfere with cleaning tasks. Competing behaviors occur when two operants are reinforced concurrently at different schedules. However, according to the matching law, the behavior that has a greater reinforcer will occur to the exclusion of the lesser reinforced behavior (Herrnstein, 1970; Neef \& Lutz, 2001).

Having to complete multiple tasks can often lead to competing behaviors. Neef and Lutz (2001) showed Ihat the quality of a reinforcer and response effort influenced which concurrent behavior students were likely to engage in. Generalized to server duties, this means that because busing may be less reinforcing, servers will engage in other behaviors that lead to more reinforcement such as chatting with other employees or attending to customers, which could result in greater gratuities. Since servers perform numerous concurrent behaviors, most of which are reinforced more often than busing, interventions such as task clarification, goal setting, and feedback might displace natural reinforcing schedules by providing more-effective contingencies for busing behaviors.

Feedback has been used widely as reinforcement (Alvero. Bucklin. \& Austin, 2001). Feedback can be administered verbally (Austin. Weatherly, \& Gravina, 2005). as well as in graphic form (Ludwig, Biggs, Wagner, $\&$ Geller, 2002). Feedback in the form of positive reinforcement has been shown to be most effective when it is directly tied to a behavior, delivered quickly, and coupled with goal setting (Ludwig \& Geller. 1991. 1997, 2000; Stephens \& Ludwig, 2005; Sulzer-Azaroff \& Mayer, 1991).

An antecedent technique designed to clarify and prompt specific behavior is called task clarification. Task clarification and verbal feedback have been jointly used to increase correct closing-time behaviors and cleaning behaviors in a university bar (Anderson, Crowell, Hantula, \& Siroky, 1988), grocery stores (Shier, Rae, \& Austin, 2003), and restaurants (Austin, Weatherly, \& Gravina. 2005). These studies indicate that cleaning behaviors can be heavily influenced by preceding feedback with task-specific information.

The current study targeted busing times at a high-volume pizza restaurant. First, researchers investigated the use of task clarification to improve busing behaviors, and then coupled task clarification with goal setting as well as weekly visual and verbal feedback on busing behaviors using an $A B C$ design. The researchers believed that, although task clarification can initially change behavior, a consequence needed to be 
paired with task clarification to reduce overall table busing times. By implementing a second intervention, tying consequences to the busing behavior (e.g., verbal and weekly graphic feedback), overall busing time was expected to be substantially reduced.

\section{METHOD}

\section{Participants and Setting}

Participants included four female lunchtime servers, ages 18-24, and one 28-year-old male manager from a high-volume pizza restaurant employed throughout the first three phases of the study. Servers were paid \$2.15 per hour on a biweekly basis; the majority of their wages were comprised of tips. One server was assigned to each section of the restaurant. Servers were responsible for seating patrons, taking orders, and delivering food. Servers were also responsible for clearing away unused dishes, plates, and so on, as customers finished their meals (i.e., prebusing). In addition to tasks involving direct interaction with patrons, servers were also responsible for busing tables once patrons left.

To bus a table, servers stacked remaining dishes, sprayed tables and chairs with a cleaning solution, completely wiped off surfaces, and took dishes back to the washroom. Although each server was assigned to just one section, servers were asked to bus tables in other sections when opportune (i.e., cross-busing). After busing a table, servers washed their own dishes. Because servers both bused tables and washed dishes, it was difficult for servers to bus tables quickly; the more they bused, the more dishes they had to wash. An employee handbook outlined written instructions on how to properly wait tables. Procedures for how to bus, prebus, or cross-bus tables were not detailed in the employee handbook.

\section{Dependent Variables}

An ABC analysis (Daniels \& Daniels. 2004) and a modified Performance Diagnostic Checklist (PDC) (Austin, 2(XX)) helped to pinpoint specific antecedents, behaviors, and consequences for this investigation. Busing time was defined as the amount of time it took a server to correctly bus a table. Data collection sessions were 30 minutes long, three days per week, and between the hours of 12:30 p.m. and 1:00 p.m. Researchers sat at a table at the side of the restaurant and coded servers by sections. To time the busing behaviors, one researcher used a Timex ${ }^{\wedge}$ Expedition wristwatch with chronological timing and lap features. The other researcher used a Nokia^" model cellular phone, which also had chronological timing and lap features.

|Researchers started to time when the last patron at the table left the table. After researchers started the initial timing, the lap feature was used to record the interval it took the server to initially arrive at the table in 
need of busing (i.e., "time to table"). Another interval was measured from the arrival at the table to the completion of busing (i.e., "time to finsh"). Timing stopped when the table was completely bused and the server walked away (i.e.. "total time"). Tables were completely bused when servers removed all of the dishes, sprayed and wiped down the tables and chairs, and replaced the napkins and salt and pepper shakers.

Researchers also recorded instances where a server prebused a table (i.e., cleaned a table while patrons were still using it), or cross-bused another server's table (i.e., a server from one section helped a server of another section bus a table).

\section{Design}

The research design used to evaluate the effects of the treatment for busing time was an ABC design. Baseline data were collected for 4 weeks. After achieving a stable baseline, researchers implemented the task clarification and goal setting in the form of a memo. Two weeks later a system of verbal and graphic managerial feedback was implemented. After an additional 4 weeks all intervention materials were removed and manager feedback was withdrawn. After a hiatus of 8 weeks where no data collection occurred, 2 weeks of follow-up data collection was conducted on a newly hired set of servers.

\section{Intervention}

Task clarification memo and goal setting. In the first phase of the intervention a memo was passed out to all employees detailing the steps necessary to correctly bus a table. In the memo, the management team set a goal to reduce the overall table busing time to 3 minutes or less (180 seconds). This goal was made explicit to employees not only at the time of receiving the memo but also throughout the verbal and graphic feedback phases. Each employee signed the memo to indicate that he or she had received and understood the instructions. During the task clarification stage, management was specifically instructed not to offer verbal or graphic feedback outside of what would normally be given to the servers busing tables.

Individual verbal and graphic feedback. Two weeks later, individual feedback was delivered weekly by the manager to individual servers on the morning of their last shift of the work week. Though the manager told employees that he watched their busing, the data for the feedback were based solely on the busing data collected by the researchers for the week. The manager reminded servers of the less-than-3-minute goal and then showed the server a graph of his or her average bus time for the week. Employees' busing times for the week were averaged and plotted in a time series across weeks. The manager verbalized if servers were above or below the average busing time for the week. He also delivered positive 
verbal feedback to servers who maintained a goal of busing tables in 3 minutes or less. Finally, the manager provided suggestions on how to reduce busing time if the server was over the 3-minute goal.

Group feedback. Weekly group feedback was posted on a large graph in the back of the restaurant at the end of each work week. Data points on the chart represented the average bus time across all servers for the preceding week. A goal line was drawn horizontally at the 3-minute mark, and data points were plotted according to the average group busing time for the week. The name of the server who had the overall best busing time over the course of the week was also posted.

\section{RESULTS}

Busing times were divided into three categories: total time, time to table, and time to finish. There were a total of 45 observations of busing over î 0 data collection sessions in baseline, 30 observations over 6 sessions during the task clarification and goal setting phase, and 28 observations over 9 sessions during the feedback phase. There were 26 observations conducted over 5 sessions during follow-up observations. Interobserver reliability was taken for 40 busing observations out of the 130 total observations (31\%). The average difference between the primary and secondary observer was 1.7 seconds, and 36 observations were within at least 3 seconds of each other. A correlation of $r=0.99$ was calculated between the primary and secondary observers' recorded busing times.

Figure I shows each of the average group busing times across each phase. Figure 2 shows the observed busing times for each server in this study over the course of the four phases. Average total time was 314.64 seconds $(S D=195.94)$ for baseline, 283.53 seconds $(S D=230.20)$ for the task clarification phase, and 151.60 seconds $\{S D=112.26)$ for the feedback phase. The average total time for follow-up was 275.80 seconds $(5 \mathrm{D}=105.86)$.

Mean time-to-table was recorded and aggregated across phases. Throughout the baseline phase, servers took an average 133.32 seconds $(S D=77.12)$ to arrive at the table and start busing. During the task clarification phase, servers arrived at the table after an average 69.00 seconds $(S D=54.36)$. During the feedback phase, servers arrived at the table after an average 62.88 seconds $(S D=57.40)$. 
FIGURE 1. Group Average Busing Time. Each data point represents an average of all participants for each session. The black points represent where public and private feedback was given.

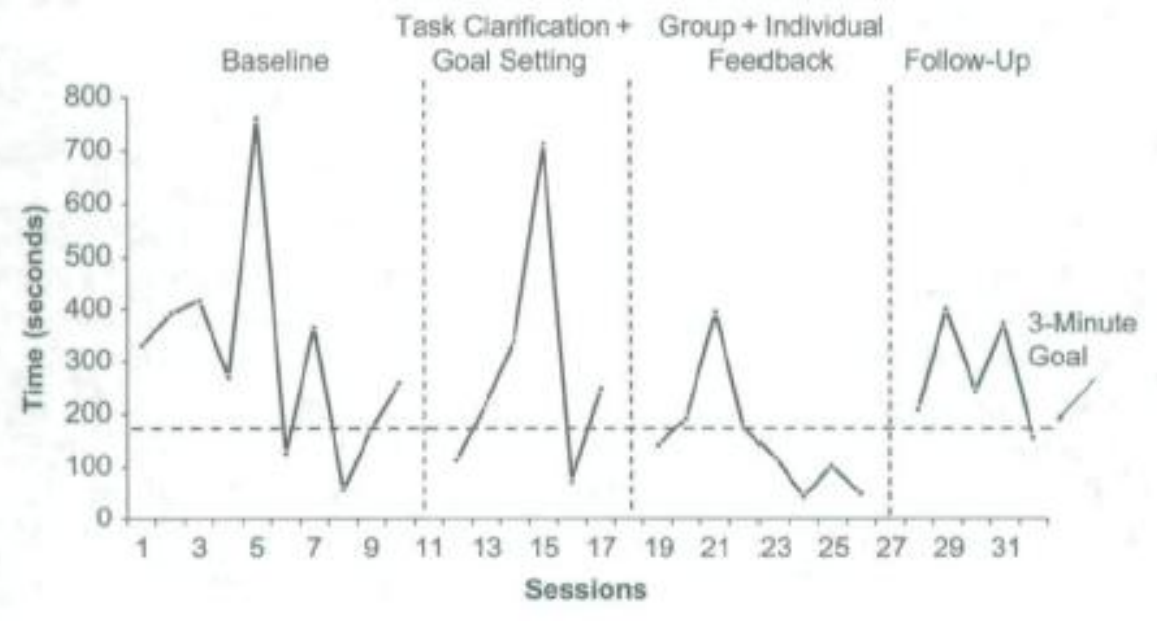

FIGURE 2. Individual Participant Busing Times. Each data point represents a table that was bused. They are in a time sequence. Each "P" means that the tabie was prebused and each "C" means that the table was cross-bused.

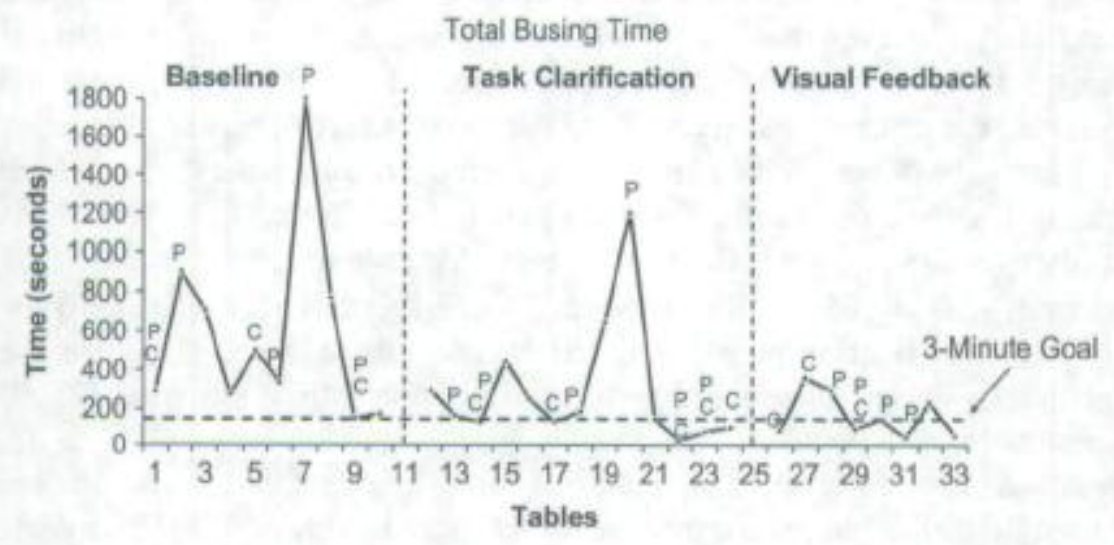


Once the server arrived at the table, researchers calculated how long it took him or her to completely bus the table. During the baseline phase, servers took an average of 181.32 seconds $(S D=189.30)$ to bus after making the initial stop at the table. For the task clarification phase, servers took 214.53 seconds $\{S D=234.85$ ) on average to completely bus the table. In the feedback phase, servers took only 88.73 seconds on average $(S D=96.46)$ to completely bus the tables. Table I details the total bus time when instances of prebusing and cross-busing occurred.

\section{DISCUSSION}

The purpose of the current research was to demonstrate how task clarification, goal setting, and employee feedback could be used to improve busing behaviors in a restaurant. Before the interventions, bus times were averaging over 5 minutes. Upon completion of both task clarification and feedback phases, busing behaviors had been improved to an average daily time of 2.5 minutes; this was under the stated management goal of 3 minutes.

During the task clarification phase, the memo explicitly outlined the behavioral components of how and when to bus a table, as well as described the goal of busing tables in less than 3 minutes. As early as the 


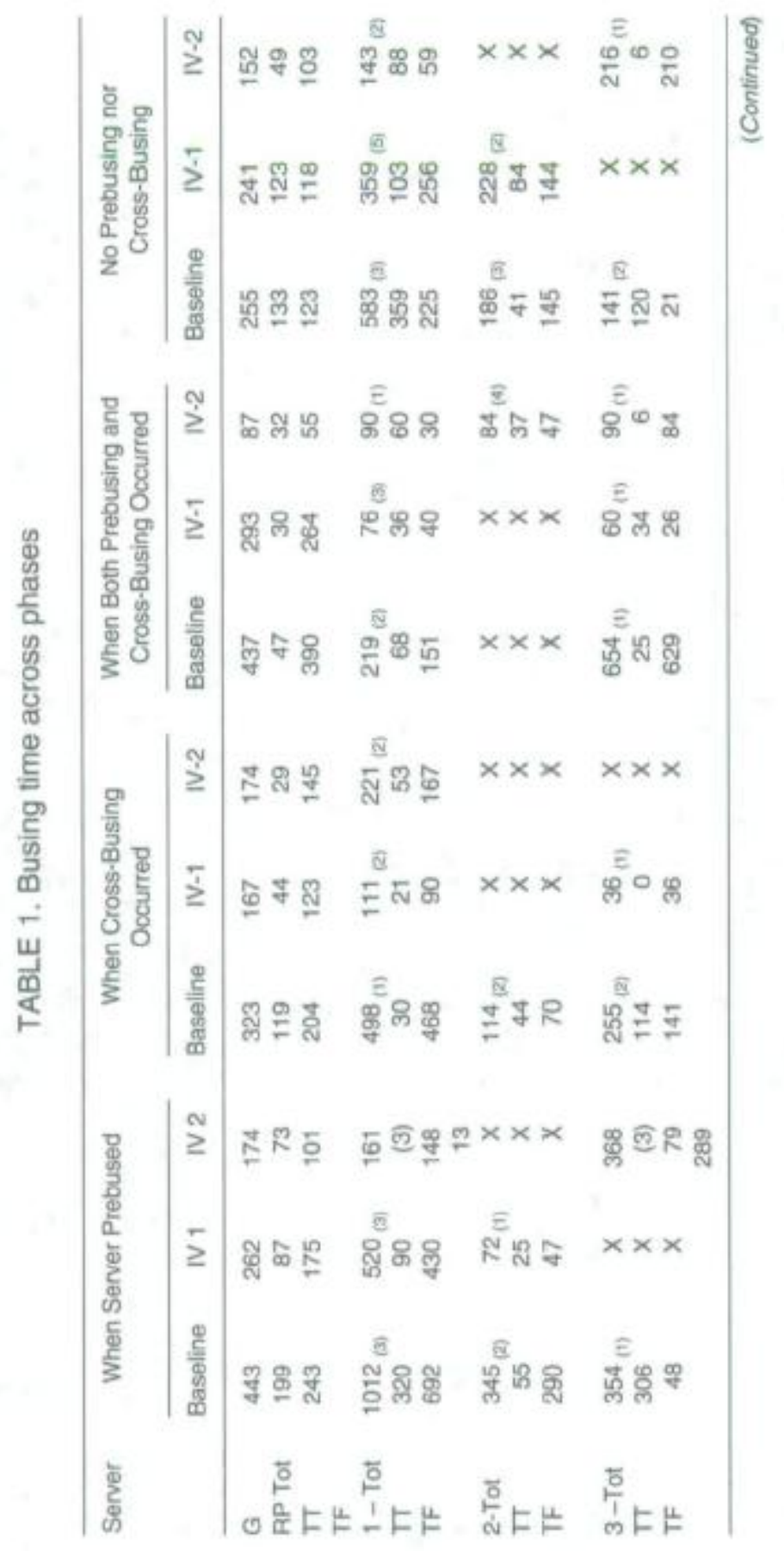




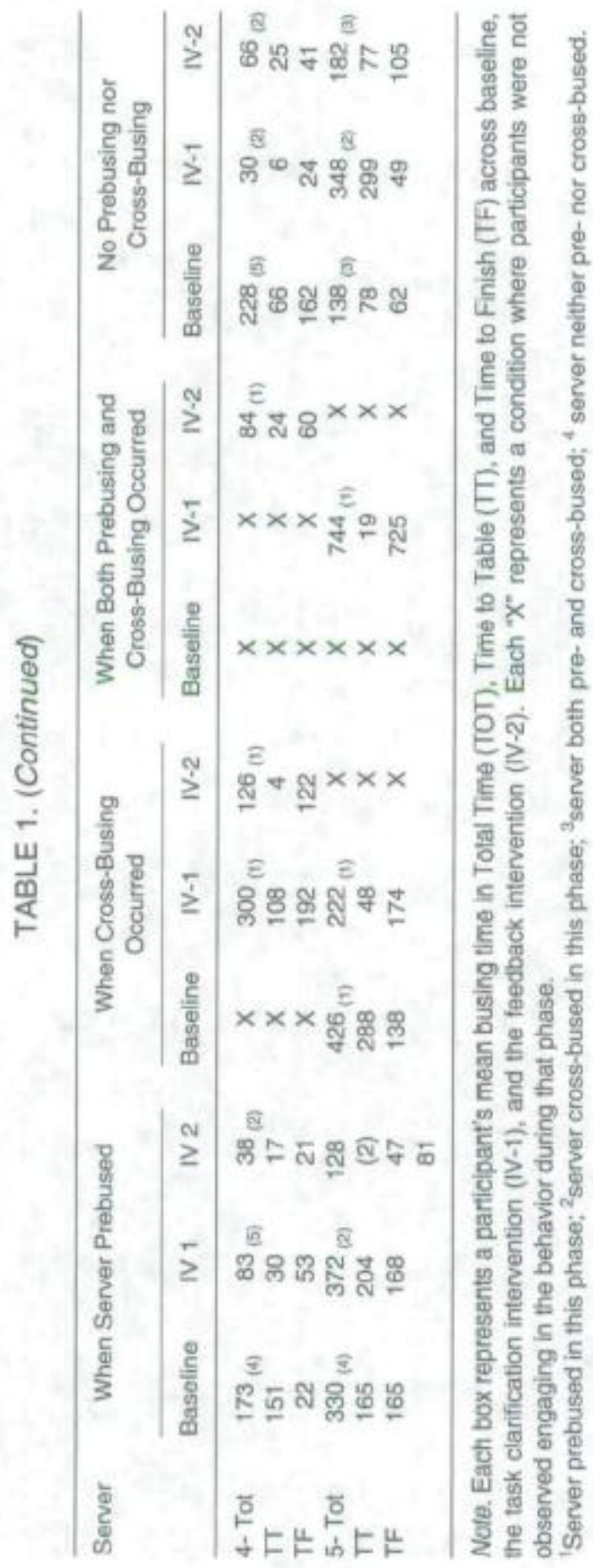

first day after the memo was circulated, researchers recorded an increase in busing behaviors consistent with those in the task clarification memo. For example, servers began taking all dishes back to the kitchen in one trip and wiping off their dirty tables before attending to other patrons.

The feedback phase was designed to provide the necessary reinforcement by having the manager provide both verbal and graphic feedback. 
Consistent with other reports on verbal and graphic feedback (Alvero, Bucklin, \& Austin, 2001; Austin, Weatherly, \& Gravina, 2005). the current research showed that after feedback bus times reduced to an average of just over 2.5 minutes. When feedback was removed in the follow-up condition, busing times reversed to near-baseline levels possibly demonstrating the functional control of the intervention operations.

An interesting relationship was observed between how long servers took to start busing a table (i.e., time to table), and when the table was finished being bused (i.e., time to finish). During the task clarification phase, the total time to bus was largely influenced by the time it took for the server to begin busing. The task clarification intervention may not have improved busing behaviors, but rather increased the likelihood of servers to start busing a table in spite of other competing behaviors. The task clarification memo only described the behaviors involved with busing once servers were actually at the table. Thus, it is reasonable to guess that shorter time-to-bus times may have been due to the 3-minute goal addressed in the memo.

During the feedback phase, shorter total busing times resulted from less time to clean, as opposed to approaching the table more quickly. The feedback intervention may have been more suitable for improving the act of busing a table. It is likely that the task clarification, which preceded the feedback phase, provided the information on correct busing behavior, which eventually informed correct busing at the table. However, it took a consequence like feedback to actually reinforce these correct behaviors.

One of the noteworthy changes that the researchers observed was an increase in prebusing and cross-busing activities. Prebusing and crossbusing behaviors were both addressed in the employee memo, but as ancillary points to busing. Throughout the task clarification phase, and even more so in the feedback phases, employees became more proactive in prebusing their tables and cross-busing other servers' tables as well. While the incidence of prebusing and cross-busing increased across the interventions, they did not consistently decrease total busing time across interventions.

\section{Limitations and Future Research}

Some limitations of the current research should be noted. First, each server was not represented equally in the group data because servers worked variable days and shifts. Second, verbal feedback was neither standardized nor verified. Feedback words and phrases were discussed with the manager at the beginning of the phase; however, researchers never actually heard management deliver feedback. Management did assure researchers that feedback was being delivered correctly on a weekly basis. Future research should establish feedback guidelines and validity checks. 
Third, goal setting was not separated from feedback during the second intervention phase leaving it undetermined which piece of the intervention created differences in busing times. Further, while pre- and cross-busing behaviors were measured, the behaviors were not given specific goals during the second intervention phase. The absence of these goals may have contributed to these behaviors not making a serious impact on busing times.

Finally, this study was originally intended to be an ABCA reversal design. However, after the second intervention phase, the entire waitstaff and the manager ceased working for various reasons. Therefore, data collected during the final phase could not serve as a return to baseline. Future research should consider multiple locations in order to achieve control.

Whereas the suggestions entailed herein would strengthen conclusions drawn by the researchers, the study does have practical value for managers. In businesses where off-task behaviors are often more rewarding than on-task behavior, and where managers have the opportunity to interact directly with employees, an intervention by management as simple as clarifying employee roles and administering consistent, accurate feedback on performance could substantially decrease competing behaviors. The research also shows that monetary or tangible incentives do not always have to be the go-to incentive to improve employee performance; constructive and/or affirming feedback can be as effective a remedy if employees know what is expected of them.

\section{REFERENCES}

Alvero, A. M.. Bucklin. B. R.. \& Austin. J. (2001 ). An objective review of the effectiveness and essential characteristics of performance feedback in organizational settings. Journal of Organizational Behavior Management, 21(1), 3-30.

Anderson D. C . Crowell C. R., Hantula D. A., \& Siroky L. M. (1988). Task clarification and individual performance posting for improving cleaning in a student-managed university bar. Joumal of Organizational Behavior Management. 9(2), 73-90.

Austin. J. (2000). The use of the performance diagnostic checklist lo guide intervention selection in an independently owned coffee shop. Journal of Organizational Behavior Management. 23\{2/3). 5-19.

Austin. J.. Weatherly, N. L.. \& Gravina, N. E. (2005). Using task clarification, graphic feedback, and verbal feedback to increase closing-task completion in a privately owned restaurant. Journal of Applied Behavior Analysis, 38(1), 117-120.

Daniels, A. C. \& Daniels, J. E. (2004) Performance management: Changing behavior that drives organizational effectiveness. Atlanta: Aubrey Daniels International.

Hermstein, R. J. (1970). On the law of effect. Journal of the Experimental Analysis of Behavior. 13(2). 243-266. 
Ludwig. T. D., Biggs, J., Wagner. S.. \& Geller, E. S. (2002). Using public feedback and competitive rewards to increase the safe driving behaviors of pizza deliverers. Joumal of Organizational Behavior Management, 21(4), 75-104.

Ludwig, T. D.. \& Geller, E. S. (1991). Improving the driving practices of pizza deliverers: Potential moderating effects of age and driving record. Journal of Applied Behavior Analysis. 24(1), 31-44.

Ludwig, T, D.. \& Geller. E. S. (1997). Assigned versus participatory goal setting and response generalization: Managing injury control among professional pizza deliverers. Journal of Applied Psychology. 82\{2), 253-261.

Ludwig. T. D., \& Geller, E. S. (2000). Intervening to improve the safety of delivery drivers: A systematic behavioral approach. Monograph. Journal of Organizational Behavior Management. 19(4). 1-124.

Neef. N. A., \& Lutz, M. (2001). Assessment of variables affecting choice and application to classroom interventions. School Psychology Quarterly. 16, 239-252.

Shier. L.. Rae, C. \& Austin, J. A. (2003). Using task clarification, checklists and performance feedback to improve the appearance of a grocery store. Performance Improvement Quarterly, 16(2), 26-40.

Stephens, S. D., \& Ludwig, T. D. (2005). Improving anesthesia nurse compliance with universal precautions using group goals and public feedback. Journal of Organizational Behavior Management. 25(2). 37-70.

Sulzer-Azaroff. B., \& Mayer, R. G. (1991). Behavior analysis for lasting change. Fort Worth, TX: Harcourt Brace. 